\title{
Biologische und biotechnologische Verfahren gegen holzbewohnende Pilze - Eine Übersicht
}

\author{
E. Graf
}

Biologische und biotechnologische Verfahren sind zwei der Aspekte des integrierten Holzschutzes. Zahlreich sind die Veröffentlichungen, welche über Möglichkeiten und Grenzen dieser Verfahren Auskunft geben (Graf 1997). Notwendige Voraussetzungen für eine Marktchance von antagonistischen Mikroorganismen oder ihrer Antibiotika sind Wirksamkeit, Human- und Umweltverträglichkeit sowie Wirtschaftlichkeit von Produktion und praktischem Einsatz. Am aussichtsreichsten dürfte die Anwendung zum Schutz von saftfrischem Rund- und Schnittholz gegen Verblauung sowie eventuell von Holzbauteilen in der Gefährdungsklasse 3 gegen holzzerstörende Pilze sein. Ein interessanter Aspekt könnte auch die bekämpfende Behandlung von geschädigtem Holz sein mit dem Ziel, einen bestehenden Pilzbefall in seiner Ausbreitung zu verzögern, um die Gebrauchsdauer eines Holzbauteils für eine bestimmte limitierte Zeit zu verlängern. Die publizierten Daten zeigen jedoch, dass noch sehr viel Grundlagenforschung geleistet werden muss. Die Wirkmechanismen müssen verstanden werden, damit ein anerkanntes, den bestehenden Gesetzen und Richtlinien entsprechendes Produkt auf den Markt gebracht werden kann.

\section{Biological and biotechnological preservation procedures against fungi present in wood $-A$ review}

Biological and biotechnological procedures are two of the aspects of integral wood preservation. Many publications report on possibilities and limits of such procedures (Graf 1997). Essential conditions for commercial application of antagonistic microorganisms or derived antibiotics are effectiveness and human or environmental compatibility as well as economic production and practical application. Most promising application can be expected in preservation of green roundwood and timber against blue staining fungi or preservation of structural timber (risc class 3 ) against wood degrading fungi. Another interesting possibility may be the treatment of already damaged wood in order to inhibit further attack and thus extend the life-time of a structural components for a limited period of time. The data reported in this review, however, reveal that

\section{Erwin Graf}

Eidgenössische Materialprüfungs- und Forschungsanstalt (EMPA), Abt. Biologie, Lerchenfeldstr. 5, 9014 St. Gallen e-mail: erwin.graf@empa.ch

Eine ausführliche Bibliographie zum Thema kann gemeinsam mit dem internen Bericht EMPA $120^{\prime} 500$ beim Autor bestellt werden much basic research is still needed. The chemical and biological mechanisms have to be understood, before a reliable product complying to existing laws and standards can be introduced on the market.

\section{1}

\section{Einleitung}

Verschiedenste Pilze können bei einer Holzfeuchte von mehr als $25 \%$ zu beachtlichen Schäden führen sei es durch sicherheitsrelevante Holzzerstörung durch Braun-, Weißoder Moderfäule, durch Bildung hygienisch bedenklicher Schimmelrasen auf Holzoberflächen (Allergiepotential) oder durch ästhetisch nicht tolerierbare Vergrauung resp. Verblauung von Rund-, Schnitt- oder Bauholz. Von ca. 1945 bis zur Ölkrise 1975 wurde versucht, viele architektonische Planungs- und Ausführungsfehler mit Hilfe chemischer Holzschutzmittel zu beheben. Doch seit dem Erscheinen des Buches "Der stumme Frühling" von Carson (1962) ist der Glaube an die Lösbarkeit aller Probleme des Material-, Pflanzen- und Vorratsschutzes mit Hilfe chemischer Produkte mehr und mehr einer kritischen, gesamtökologischen Betrachtungsweise gewichen. Vermehrt wurde versucht, Pilzschäden im Bauwesen durch architektonische Gestaltung und durch konstruktive Lösungen zu verhindern. Parallel zur zunehmenden Ablehung "chemischer" Produkte breitete sich aber auch ein unreflektierter Glaube an einen problemfreien Holzschutz mit "biologischen" Erzeugnissen aus.

Ziel der folgenden Ausführungen ist, die Möglichkeiten und Grenzen biologischer und biotechnologischer Verfahren zum Schutz des Holzes gegen Pilze in einen Gesamtzusammenhang des integrierten Holzschutzes zu stellen.

\section{2}

Unter integriertem Holzschutz (Bild 1) versteht man die optimale Kombination verschiedener potentieller Schutzmaßnahmen zu einem der jeweiligen Fragestellung angepassten Schutzkonzept. Die Festlegung des Maßnahmenpakets muss dabei auf der Wirksamkeit des Konzepts, der Sicherheit der Konstruktion, der Gesundheit des Menschen, der Umweltverträglichkeit sowie der wirtschaftlichen Realisierbarkeit der verschiedensten möglichen Maßnahmen (äußerster Kreis) basieren. Ziel ist, so in das Ökosystem einzugreifen, dass sich holzschädigende Organismen nicht mehr vermehren können resp. ihre Ausbreitung verzögert wird. Das Ökosystem holzzerstörender Organismen (Pilze, Insekten) ist in seinem innern 


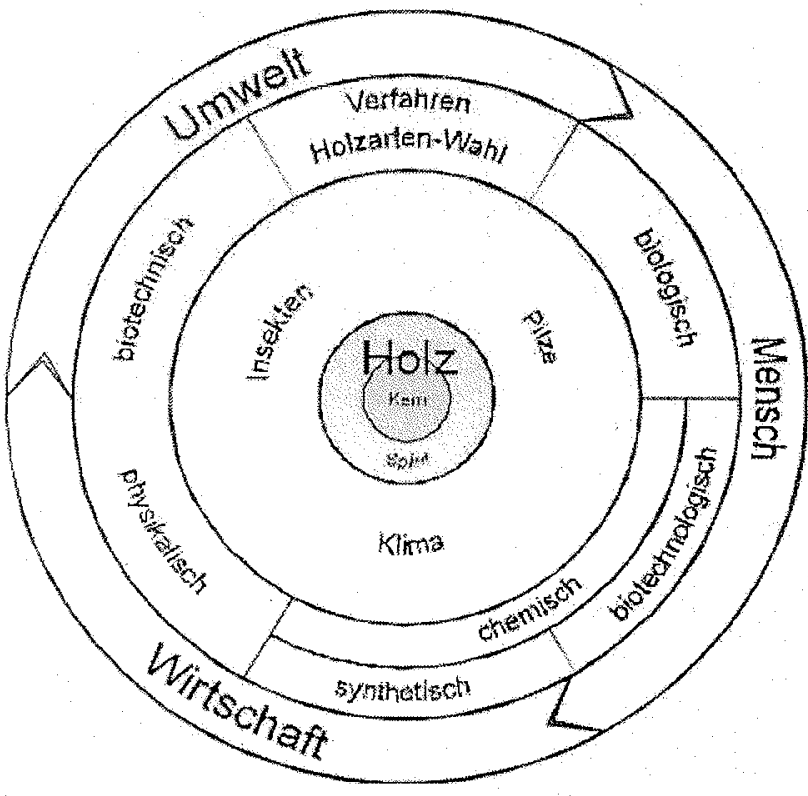

Bild 1. Integrierter Holzschutz

Fig. 1. Integral wood preservation

Kreis primär geprägt durch die Nahrung (Holzart und Holzbestandteile), das Mikroklima (Holzfeuchte und Temperatur) sowie durch die konkur renzierenden Organismen und Viren. Dieses System kann prinzipiell durch die Holzartenwahl, durch physikalisch-konstruktive, durch biotechnische (Fallen sowie Eingriff ins Erbgut der Schädlinge), durch biologische sowie chemische Maßnahmen (synthetisch oder biotechnologisch hergestellte Produkte) beeinflusst werden.

Biologische und biotechnologische Verfahren sind somit zwei der verschiedenen Aspekte des integrierten Holzschutzes (Tabelle 1). Die beiden Begriffe stammen aus dem Pflanzen- und Vorratsschutz (Franz 1972) und sind somit im Holzschutz nicht mehr frei definierbar, wie dies in verschiedensten Veröffentlichungen und Zeitschriften zum Holzschutz immer wieder geschieht. Unter biologischen Verfahren versteht man die Bekämpfung von Orga-' nismen mit Organismen, das heißt Einsatz von Antagonisten wie von Räubern, Parasiten und Krankeitserregern (Pilze, Bakterien, Viren). Auf das angesprochene Thema bezogen bedeutet dies die Bekämpfung holz- zerstörender und -verfärbender Pilze durch Pilze oder Bakterien.

Biotechnologische Verfahren umfassen die industrielle Produktion oder Extraktion von Antibiotika aus Pflanzen und Mikroorganismen, welche zum Schutz gegen biogene Schäden oder zum Abstoppen einer aktuellen Zerstörung auf oder in das Holz gebracht werden können. Es handelt sich dabei um interne Abwehrstoffe oder um Ausscheidungsprodukte wie Enzyme, Toxine, Antibiotika. Dies sind biochemische Substanzen, welche sich primär durch das Produktionsverfahren mit Hilfe von Mikroorganismen von industriell synthetisierten Stoffen unterscheiden (Bild 1). Bei einem biotechnischen Verfahren entstehen verschiedenste Substanzen, aus welchen der gesuchte Wirkstoff durch diverse Trenn- und Reinigungsverfahren herausisoliert werden muss.

\section{Biologische Verfahren}

Hier stellt sich die Frage, ob in der Natur antagonistisch wirksame Mikroorganismen (Bakterien oder Pilze) existieren, welche Holz schadfrei besiedeln und gleichzeitig einen Bewuchs durch holzzerstörende und/oder -verfärbende Pilze verhindern (Bioprotection) oder aber einen bereits bestehenden Befall abstoppen (Biocontrol) können (Freitag 1991). Mögliche Mechanismen sind die sehr verbreitete Nahrungskonkurrenz, der Mykoparasitismus, bei welchem der Nutzpilz in die Pilzfäden (Hyphen) des Schadpilzes eindringt und mit Hilfe von Enzymen die Zellwände auföst, oder die Bildung gegen Schadpilze giftiger Antibiotika (Baker 1968).

Der erfolgreiche Einsatz von Nutzpilzen und -bakterien setzt voraus, dass diese Organismen

im anvisierten Einsatzgebiet während der geforderten Zeitperiode je nach Anwendungsbereich 6 Monate bis 30 Jahre alle holzverfärbenden und/oder -zerstörenden Pilze dominieren und keine Selektivität auf nur einzelne bläue- oder fäuleerregende Pilze aufweisen.

nicht durch eine selektive Wirkung auf einen einzelnen oder eine Gruppe von Schadpilzen die Entwicklung anderer holzschädigender Pilze fördern.

sich nur von einfachen Zellinhaltsstoffen ernähren und selbst oder durch ihre Ausscheidungsprodukte weder die strukturbildende Lignozellulose-Matrix angreifen noch das Holz verfärben; im Unterschied zur Landund Forstwirtschaft gibt es im Holzschutz keine

Tabelle 1. Differenzierung von biologischem und biotechnologischem Holzschutz

Table 1. Differentiation between biological and biotechnological wood preservation

\begin{tabular}{|c|c|c|c|c|}
\hline \multirow[t]{2}{*}{ Aktives Prinzip } & \multirow[t]{2}{*}{ Verfahren } & \multirow{2}{*}{$\begin{array}{l}\text { Reinheit der } \\
\text { Rohstoffe }\end{array}$} & \multicolumn{2}{|l|}{ Wirkung gegen } \\
\hline & & & Insekten & Pilze \\
\hline Organismen & biologisch & keine & Chrysanthema & Trichoderma Scytalidium Gliocladium \\
\hline $\begin{array}{l}\text { Chemische Stoffe } \\
\text { Enzyme, Antbiotika, } \\
\text { Ausscheidungsprodukte } \\
\text { Syntheseprodukt }\end{array}$ & $\begin{array}{l}\text { biotechnologisch } \\
\text { chemisch synthetisch }\end{array}$ & $\begin{array}{l}\text { mittel } \\
\text { hoch }\end{array}$ & $\begin{array}{l}\text { Pyrethrum } \\
\text { Permethrin }\end{array}$ & $\begin{array}{l}\text { 6-Pentyl-a-pyron } \\
\text { Chitinase Scytalidin Gliovirin } \\
\text {... }\end{array}$ \\
\hline
\end{tabular}


Schadenschwelle, sondern es wird eine Nulltoleranz gefordert.

tief ins Holz eindringen und alle Bereiche des Holzes homogen besiedeln.

stresstolerant sind, das heißt genügsamer sind bezüglich Nahrungs- und Feuchteanspruch sowie widerstandsfähiger gegen Hitze und Trockenheit als die Schadpilze.

für den Langzeitschutz ein Dauerwachstum zeigen, Dauersporen bilden und/oder chemisch und biologisch stabile toxische Metaboliten produzieren können, welche der Überbrückung von Trockenperioden dienen. weder auf den Anwender von Bioprodukten noch auf den Bearbeiter und Verwender des behandelten Holzes toxisch wirken, noch direkt über Sporen oder indirekt über pilzfressende Milben (Acarina) und Staubläuse (Psocoptera) Allergien hervorrufen je nach Gesetzgebung verschiedener Länder weder genmanipuliert (z.B. Schweiz) noch biotopfremd (Deutschland) sind. In Bezug auf die Anwendung vor allem von gentechnisch veränderten und/oder pathogenen Organismen ist in geschlossenen Systemen die EU-Richtlinie 90/219/ EWG und bezüglich Freisetzung und Inverkehrbringen die EU-Richtlinie 90/220/EWG zu berücksichtigen. Für Feldforschung mit diesen Organismen bedarf es in der Schweiz einer Bewilligung durch die Bundesbehörde.

weder Haftprobleme bei Anstrichen zur Folge haben noch die Entwicklung eines sekundären Befalls durch holzzerstörende Insekten fördern (z.B. Anobien).

Die meisten Forschungsarbeiten wurden während der Jahre 1970 bis 1990 publiziert. Seit diesem Zeitpunkt ist die Zahl der Publikationen stark rückläufig (Graf 1997). Zu Beginn wurde vor allem im Labor nach antagonistisch wirksamen Mikroorganismen gesucht. Aufgrund der Misserfolge resp. der nicht reproduzierbaren Daten in Freilandversuchen liegt der Schwerpunkt der Forschung darauf, in Laborversuchen die antagonistischen Mechanismen und die Bedingungen für eine erfolgreiche Anwendung von Mikroorganismen zum vorbeugenden Schutz gegen Verblauung und Verschimmelung von saftfrischem Holz (1) und gegen Besiedlung und Zerstörung von gelagertem resp. verbautem Holz (2) sowie zur Bekämpfung eines bestehenden Befalls in konstruktiven Holzbauteilen (3) abzuklären.

\section{1}

\section{Wirkung gegen holzverfärbende Pilze beim saftfrischen Schnittholz}

Zum Schutz von saftfrischem resp. abtrocknendem Schnittholz gegen Sekundärbläuepilze wird eine Wirkungsdauer von maximal 6 Monaten verlangt, bis die Holzfeuchte bei $18 \%$ und tiefer liegt. Pilze wie Acremonium strictum, Byssochlamis nivea, Penicillium thomii und Stilbella aciculosa, Clitocybe geotropa, Scytalidium, Trichoderma und Gliocladium zeigen eine bestimmte Hemmwirkung, besonders wenn sie auf keimarmem Holz vorinkubiert werden. In den USA wird von der Papierindustrie bei der Holzschnitzellagerung eine weiße Mutante des Bläuepilzes Ophiostoma piliferum (Cartapip 97®) zum
Abbau des Harzanteils des Holzes eingesetzt. Schmidt und Müller (1996) wiesen nach, dass dieser Pilz die Verblauung des Holzes reduzieren kann.

Von den Bakterien erwiesen sich Bacillus subtilis und Arten der Gattungen Pseudomonas (z.B. Pseudomanas cepacia) und Streptomyces am wirksamsten (Graf 1990). Da $B$. subtilis weder eine wirksame Menge Antibiotika bilden noch die Sporenkeimung verhindern kann, wird mit keinem vollständigen Schutz gerechnet (Kreber 1993). Ein limitierender Faktor beim Einsatz von Bakterien ist, dass diese in der Regel eine höhere Wasseraktivität des Materials verlangen als Pilze und somit nicht den ganzen Wachstumsbereich der Pilze abdecken können.

Obwohl nur eine kurze Wirkungsdauer verlangt wird, haben die geprüften antagonistischen Mikroorganismen in Praxisversuchen versagt oder zu widersprüchlichen Ergebnissen geführt (Liu 1996). Bereits geringe Änderungen im Nahrungsangebot, der klimatischen Verhältnisse, der Begleitmikroflora und von Nahrungsfaktoren des Holzes beeinflussen die antibiotische Wirkung der nützlichen Pilze und Bakterien (Morrell 1992, 1995). Bläuepilze bilden in der Regel im Holz sehr schnell ihre dunklen Pigmente, so dass die durch mykoparasitische Pilze verursachte Auflösung der Zellen meist zu spät eintritt, oder das Holz müsste anschliessend chemisch gebleicht werden. Zum Teil führten die eingesetzten Nutzpilze selbst zu lokalen Verfärbungen des Holzes.

\section{2}

\section{Wirkung gegen holzzerstörende Pilze}

Seit etwa 25 Jahren wird nach geeigneten Mikroorganismen gesucht, welche eine Holzzerstörung durch Pilze verhindern können. Die beiden Actinomyceten Streptomyces violaceaniger und $S$. griseus hemmten im Laborversuch, sofern eine Vorinkubation dieser Mikroorganismen erfolgte, den Holzabbau durch Coriolus versicolor, Gloeophyllum trabeum und Chaetomium globosum (Greaves 1970). Diese Resultate wurden nicht weiter verfolgt. Am ehesten wird heute mit einem Erfolg zur Vorbeugung gegen resp. Bekämpfung von Braun- und Weißfäule erregenden Pilzen mit einem Pilz oder Pilzgemisch aus den Gattungen Gliocladium, Scytalidium und Trichoderma gerechnet. Im Laborversuch zeigt Gliocladium virens ein breites Wirkungsspektrum gegen Braunfäulepilze, die er überwächst und in den meisten Fällen abtötet. Scytalidium lignicola und S. album wirken im Laborversuch nach einer Vorinkubation gegen verschiedene Braun- und Weißfäule-Erreger und sind befähigt diffusionsfähige Antibiotika zu bilden. Verschiedene Pilzarten aus der Gattung Trichoderma sind konkurrenzfähige Saprophyten, haben mit ihren zellwandauflösenden Enzymen mykoparasitische Fähigkeiten und bilden flïchtige und nicht flüchtige antibiotische Stoffe gegen verschiedene Braunfäuleerreger (Murmanis 1988). Die Trichoderma-Arten weisen aber ein Wirkungsdefizit gegen Blättlinge (Gloeophyllum spp.) und Weißfäuleerreger auf (Highley 1988).

Um ein breites Wirkungsspektrum zu erreichen, wurden auch Gemische von Mykoparasiten getestet. Verschiedene Forschungsgruppen versuchten, durch eine künstliche Infektion mit Trichoderma und Scytalidium 
spp. eine Holzzerstörung in teerölimprägnierten Douglasien-Leitungsmasten zu bekämpfen. Bruce (1991) beimpfte Leitungsmasten mit dem Handelsprodukt BINAB FYT $®$, welches Sporen und Myzelfragmente von Trichoderma polysporum, T. harzianum und Scytalidium spp. FY enthält. Die verschiedenen Feldversuche in der Gefährdungsklasse 4 (Tabelle 2) haben im Vergleich zu den Labortests sehr variable Ergebnisse mit eher begrenztem Erfolg gezeigt. Sieben Jahre nach Versuchsansatz war Trichoderma immer noch aktiv im Holz vorhanden. Er verhinderte im Mastinnern eine weitere Ausbreitung von Lentinus lepideus und Antrodia carbonica. Der Schutz gegen Coriolus versicolor war ungenügend. In Laborversuchen von Oxley (1977) vermochten Trichoderma viride und Scytalidium lignicola einen Befall von Holzproben durch die wichtigen Braunfäuleerreger Poria placenta und Gloeophyllum trabeum nicht zu verhindern. Es wird angenommen, dass in den teerölimprägnierten Masten erst die residente Pilzflora zum Beispiel durch Hitzesterilisation oder Begasung abgetötet werden muss, um einen sicheren Erfolg mit Trichoderma zu erzielen. Diese Vorbehandlung bewirkt, dass dieses Verfahren nicht ökonomisch sein kann.

Auch bei der vorbeugenden Behandlung wird angenommen, dass zuerst die residenten Pilzflora im $\mathrm{Holz}$ desinfiziert werden muss, bevor eine wirksame Besiedlung des Holzes durch antagonistische Mikroorganismen, z.B. durch Scytalidium lignicola und Gliocladium virens, möglich ist (Giron 1989).

Nach Canessa (1996) kann der Einsatz von Trichoderma harzianum gegen Braunfäulepilze dazuführen, dass der Weißfäulepilz Coriolus versicolor, welcher in der Gefährdungsklasse 4 aktiv ist, mehr Laccase, ein Phenoloxydase-Enzym bildet, welches den Lignin-Abbau fördert. Dies kann den Abbau der freien Zellulose durch Trichoderma ermöglichen.

Messner (1995) beschränkt die Anwendung seines Holzschutzverfahrens auf die Gefährdungsklasse 3, da hier ein weniger breites Spektrum holzzerstörender Pilze - vor allem Braunfäulepilze - erwartet wird. Das Holz wird vorbeugend mit einer Suspension von Konidiosporen von Trichoderma harzianum druckimprägniert und anschlieBend unter kontrollierten Bedingungen vorbebrütet. Nach einer Versuchszeit von 3 Jahren zeigten auf Betonfüßen vertikal montierte Mastabschnitte noch keine Anzeichen eines Pilzbefalls. Es waren aber auch keine dichten Sporenrasen von Trichoderma feststellbar. Die antimykotische Wirkung basiert nach Messner (1995) auf der Bildung flüchtiger Hemmstoffe wie z.B. 6-Pentyl-a-pyron und Trichorzianin, auf lytischen Enzymen wie Chitinase und Proteasen, auf dem Entzug von Spurenmetallen durch Siderophore sowie auf der Bildung von Chlamydosporen, welche Trockenperioden überdauern können. Erste mit Sporen- und Myzelsuspensionen durchgeführte Toxizitätstests zeigten offenbar keine negativen Wirkungen. Trichoderma selb'st wurde von der Weltgesundheitsorganisation (WHO) in die Risikoklasse 1 - ohne Risiko für Menschen - eingeteilt. Da es sich hier um vertikale und nicht um horizontale Bauteile ohne Erdkontakt handelt, kann aber eine abschließende Beurteilung der biologischen Wirkung erst in einigen Jahren erfolgen.

\section{Biotechnologische Verfahren}

Grundsätzlich führen zwei Wege zu neuen Wirkstoffen: die chemische Synthese verschiedenster neuer Verbindungen und die Suche nach aktiven Naturstoffen. Bei den Naturstoffen liegt die Trefferwahrscheinlichkeit etwas höher und wegen der Komplexizität der Strukturen ist die Zahl der möglichen Metaboliten praktisch unbegrenzt. Nachteilig ist die oft schwierige und teure Herstellung und die schlechtere Zugänglichkeit der Derivate, das heißt die Produktion reiner Wirkstoffe, welche nicht verunreinigt sind durch andere Chemikalien. Als Wirkstofflieferanten können für den Holzschutz Pflanzen und Mikroorganismen dienen.

\section{1}

\section{Pflanzliche Stoffe}

Die im Vergleich zu Fichte und Tanne erhöhte Resistenz diverser Holzarten gegen holzzerstörende Pilze kann auf verschiedene antimykotische Pflanzeninhaltsstoffe zurückgeführt werden. Als wirksame Antimykotika wurden unter anderem isoliert: $\beta$ - und $\lambda$-Thujaplicin sowie $\beta$-Thujaplicinol aus Thuja plicata, $p$-Methoxythymol aus der Weihrauchzeder, Tectochinon aus Teakholz, Dihydroflavonol aus Piptadeniastrum africanum sowie amerikanisches Zedernholzöl und chinesisches Pinienöl. Da es wirtschaftlicher ist, resistente Hölzer direkt einzusetzen als zuerst die Stoffe zu extrahieren und in andere Hölzer zu imprägnieren, sind diese pflanzlichen Extrakte für den direkten Einsatz in der Praxis wohl kaum von großer wirtschaftlicher Bedeutung.

Kunz (1995) prüfte über 350 pflanzliche Rohstoffe im Labor auf ihre bläuewidrige Wirkung gegen Aureobasidium pullulans, Ceratocystis fimbriata und Pencillium

Tabelle 2. Gefährdungsklassen des Bauholzes gemäss EN 335

Table 2. Risc classes of structural timber according to EN 335

\begin{tabular}{|c|c|c|c|}
\hline Gefährdungsklasse & Feuchteniveau & Gebrauchsbedingungen & Organismen \\
\hline 0 & Trocken $(<12 \%)$ & Wohn-/Arbeitsräume & keine \\
\hline 1 & Trocken $(12-18 \%)$ & $\begin{array}{l}\text { Ohne Erdkontakt } \\
\text { Abgedeckt, trocken }\end{array}$ & Insekten \\
\hline 2 & Gelegentlich über $18 \%$ & Ohne Erdkontakt, außen unter Dach, Feuchträume & Pilze/Insekten \\
\hline 3 & häufig über $20 \%$ & $\begin{array}{l}\text { Ohne Erdkontakt; Außenbedingungen; nicht } \\
\text { abgedeckt; resp. Feucht- und Nassräume }\end{array}$ & Pilze/Insekten \\
\hline 4 & ständig nass, über $20 \%$ & mit Erd- oder Süßwasserkontakt & Pilze/Bakterien \\
\hline
\end{tabular}


glabrum. Daraus ergab sich, dass primär pflanzliche Phenole aus der Gruppe der Flavonoide und phenolische Säuren gegen Schnittholzbläue weiter erforscht werden sollten. Ob diese Stoffe eine genügende Persistenz gegen Bewitterung und photochemischen und biologischen Abbau zeigen, dass sie auch gegen Bauholzbläuepilze wie z.B. Aureobasidium pullulans im Bauwesen eingesetzt werden könnten, müsste in spezifischen kombinierten Freiland- und Laborversuchen nachgewiesen werden.

\section{2}

\section{Mikrobiogene Stoffe}

Bedingt durch sehr widersprüchliche Erfolge beim direkten Einsatz von Mikroorganismen im Holzschutz, können in Zukunft Stoffe mikrobiogenen Ursprungs direkt oder indirekt an Bedeutung gewinnen. Mit Bakterien und Pilzen können antibiotische Metaboliten mit komplexen Strukturen in großem Umfang zu beliebiger Zeit mit biotechnischen Verfahren reproduzierbar hergestellt werden. Bis heute wurden ungefähr 6'000 biologisch aktive Metaboliten aus ca. 500 Actinomyceten-Arten isoliert. Bei Basidiomyceten wird bei mehr als $10 \%$ der Arten ebenfalls mit entsprechenden Stoffwechselprodukten gerechnet. Diese Stoffe können in pilzspezifische physiologische Prozesse eingreifen und z.B. die Enzymproduktion, die Eiweiß- oder Chitinsynthese behindern, in die DNA-Synthese eingreifen oder die Zellmembranen und -strukturen schädigen.

In biotechnologischen Verfahren hergestellte Stoffe müssen für einen erfolgreichen Einsatz wie chemisch synthetisierte Substanzen folgende Voraussetzungen erfüllen:

Biologische Wirkung gegen alle im Anwendungsbereich schädlichen Holzpilze (keine selektive Wirkung nur auf eine Pilzgattung).

Gute Eindringung ins Holz.

Persistenz der Stoffe während der geforderten Wirkungsdauer gegen photochemischen und biologischen Abbau, gegen Verdunstung und Auslaugung entsprechend der Gefährdungsklasse, in welcher sie eingesetzt werden sollen. Die Persistenz ist vor allem von großer Bedeutung beim Langzeitschutz in den Gefährdungsklassen 3 und 4 im Hoch- und Tiefbau.

Möglichst keine Human- und Ökotoxizität. Dies erfordert auch eine hohe Reinheit des Stoffes.

Je nach Land nationale Zulassung des Stoffes analog zu den chemisch synthetisierten Substanzen.

Als potentielle Antimikrobika für den Holzschutz sind unter anderem aus Laborversuchen bekannt Gliovirin, Scytalidin, Hyalodendrin und Cytosporiopsin sowie Chitinase. Das Gliovirin ist ein Stoff von Gliocladium spp. mit hoher antimykotischer Wirkung. Beim Scytalidin handelt es sich um einen fungitoxischen Metaboliten von Scytalidium album. In geringen Dosierungen verhindert es die Sporenkeimung von Ceratocystis ulmi. Es ist ein Antagonist zu Poria carboniaca, Gloeophyllum trabeum und zum Bläuepilz Graphium spp.. Scytalidin weist offenbar eine gute Persistenz gegen Auslaugung auf, ist aber nicht hitzestabil. Mit der von Trichoderma harzianum und Serratia plymuthica produzierten Chitinase konnte im Labortest die Bläue eines Pilzgemisches je nach
Versuchsansatz zwischen 60-90\% reduziert werden (Liu 1996). Stark antibakteriell, antimykotisch und cytotoxisch wirken Strobilurine und Oudemansine sowie Diterpene und Striatine. Nach Messner (1995) weist Trichoderma harzianum ein ganzes Spektrum antimikrobieller Stoffe auf, so z.B. als Hemmstoffe das flüchtige 6-Pentyl-apyron und die löslichen Trichodermin, Isonithrin, Cyclosporin und Trichorzianine sowie die lytischen Enzyme Chitinase und Protease.

Bisher wurden auch diverse Kulturfiltrate auf antimykotische Wirkung geprüft, ohne anschließend die biologisch aktiven Substanzen chemisch zu charakterisieren. Unter andern erwies sich ein wässeriges Filtrat aus Trichoderma viride als entwicklungshemmend gegen verschiedene Schimmelpilze. Gegen Lentinus lepideus zeigten Trichoderma viride, Scytalidium lignicola, Gliocladium roseum, Penicillium funiculosum, Epicocum purpurascens und Fusarium solani antibiotisch wirksame Stoffwechselprodukte. Gegen Schnittholzbläue wies ein Kulturfiltrat des Bakteriums Pseudomonas cepacia eine bestimmte biologische Aktivität auf.

Doch diese Stoffe wurden bisher weder allein noch in einer Holzschutzmittelformulierung auf ihre Persisten $z$ gegen die unterschiedlichen Umwelteinfüsse in den verschiedenen Gefährdungsklassen geprüft.

\section{5}

Kritische Betrachtung biologischer und biotechnischer Verfahren

Nach Durchsicht der Holzschutzliteratur kann heute kein abschließendes Urteil über die praktische Anwendbarkeit biologischer und biotechnischer Verfahren gegeben werden. Ein Großteil der Versuche wurde nur im Labor durchgeführt. Es wurde bisher nur eine begrenzte Zahl antagonistischer Mikroorganismen untersucht. Die interaktiven Mechanismen zwischen den verschiedenen holzbesiedelnden Pilzen und Bakterien sind noch weitgehend unbekannt. Die Feldversuche zeigten z.T. keinen Erfolg oder sehr widersprüchliche Resultate. $\mathrm{Zu}$ vielen Labordaten fehlen die entsprechenden Feldversuche oder deren Versuchsdauer ist noch zu kurz, um Schlüsse ziehen zu können.

Wegen des breiten Artenspektrums von Braun-, Weißund Moderfäule erregenden Pilzen und der Schwierigkeit einer uniformen Kolonialisierung durch die Nutzorganismen in der Gefährdungsklasse 4 dürfte Holz in Erdkon takt mit Hilfe dieser Verfahren wohl kaum langfristig vorbeugend geschützt werden können (Tabelle 3 ). Die hohe Populationsdichte der übrigen nicht holzzerstörenden, residenten Pilzarten dürfte eine homogene Kolonialisi erung des Holzquerschnittes durch die Antagonisten verhindern. Weisen Nutzorganismen wie z.B. Scytalidium und Trichoderma im Feldversuch nur eine Wirkung gegen Braunfäulepilze auf, nicht aber gegen Weißfäuleerreger, so kann die Besiedlung und Zerstörung des Holzes durch diese Pilze durch die fehlende Konkurrenz gefördert werden.

Etwas höher dürften die Erfolgschancen liegen für einen vorbeugenden Einsatz antagonistischer Mikroorganismen oder der von ihnen produzierten antimikrobiellen Stoffe gegen Verblauung von saftfrischem Rund- und 
Tabelle 3. Anwendungsmöglichkeiten biologischer und biotechnischer Verfahren

Table 3. Possible applications of biological and biotechnological procedures

\begin{tabular}{|c|c|c|c|c|}
\hline Holzschutz & & Anwendungsbereiche & Güte-Label & Chancen \\
\hline \multirow[t]{5}{*}{ Vorbeugend } & temporär (bis $6 \mathrm{Mte}$ ) & Rund- und Schnittholzbläue & Bs & gross \\
\hline & mittelfristig (bis 5 Jahre) & Gefährdungsklasse 3 & $\mathrm{~B}, \mathrm{P}, \mathrm{IV}$ & offen \\
\hline & & $\begin{array}{l}\text { Fassadentäfer, temporäre Bauwerke (z.B. Not- und } \\
\text { Baubrücken) }\end{array}$ & & \\
\hline & langfristig ( 5 bis 30 Jahre) & Gefährdungsklasse 4 & $\mathrm{P}, \mathrm{IV}, \mathrm{W}, \mathrm{E}$ & gering \\
\hline & & $\begin{array}{l}\text { Leitungsmasten, Lawinen- und Geländeverbauungen, } \\
\text { Sicht- und Schallschutzwände, Palisaden, Spielgeräte }\end{array}$ & & \\
\hline Bekämpfend & temporär & Fenster, Pergolen, temporäre Bauwerke & $\mathrm{Pb}$ & mittel \\
\hline
\end{tabular}

Schnittholz. In diesem Anwendungsbereich wird eine Wirkung von maximal 6 Monaten gefordert. Von großer Bedeutung beim biologischen Schutz gegen Schnittholzbläue dürfte sein, ob Holzbearbeiter und Bauherren in Zukunft eine lokale oder schwache Verblauung anstelle einer Nulltoleranz akzeptieren werden. Sonst könnten hier neben chemisch synthetisierten Stoffen biotechnologisch hergestellte Antibiotika mit niedrigem Dampfdruck, welche während 6 Monaten gegen Verdunstung sowie gegen photochemischen und biologischen Abbau resistent sind, die Mittel der Wahl sein. Soll das Holz zum Schutz gegen Verblauung mit antagonistischen Pilzen behandelt werden ist es wichtig, dass diese während ihrer Wachstumsphase möglichst keine allergene Sporen bilden und bei den Verarbeitern des behandelten Schnittholzes keine Allergien auslösen können.

Über einen möglichen langfristigen Erfolg in der Gefährdungsklasse 3 kann anhand der bestehenden Literatur noch keine sichere Aussage gemacht werden. Sollte eine langfristige wirksame Blockierung holzzerstörender Pilze nach Druckimprägnierung des Holzes mit lebenden antagonistischen Mikroorganismen an vertikal und horizontal wetterexponierten Holzbauteilen ohne Erdkontakt (Gefährdungsklasse 3) möglich sein, hätte dies den Vorteil, dass durch das stete Wachstum der Nutzorganismen und durch das Überleben wachstumsungünstiger Perioden in Sporenform ein Schutz erreicht werden könnte, der länger dauern würde als eine chemische Behandlung mit organischen Fungiziden, besonders wenn diese nur in einem Oberfl̈̈chenverfahren appliziert werden. Kritisch dürften in diesem Anwendungsbereich u.a. die homogene Verteilung einer hohen, konkurrenzlosen Konzentration des oder der Nutzpilze im Holz sowie das geforderte Nahrungsangebot sein. Die Nahrung liegt nicht in allen Hölzern in gleichem Maße und gleicher Zusammensetzung noch in großen Mengen vor. Andrerseits dürfen - um die Struktur des Holzes nicht zu beeinträchtigen - außer den Zellinhaltsstoffen weder Zellulose, Hemizellulose noch Lignin abgebaut werden. Erste Versuche zur Anreicherung des Holzes mit spezifischen Nährstoffen haben gezeigt, dass die holzzerstörenden Pilze dadurch mehr gefördert werden als ihre Antagonisten (Morrell 1990). Muss vor einer Beimpfung des Bauholzes die residente Pilzflora desinfiziert werden, wie Bruce (1991) fordert, so muss dies in die Wirtschaftlichkeitsberechnung des biologischen Verfahrens einfließen. Es könnte aber auch sein, dass durch eine Kombination traditioneller Holzschutzmittel mit Mikroorganismen die Konzentration des chemischen Wirkstoffes gesenkt werden könnte.

Bei tragenden Holzbauteilen in den Gefährdungsklassen 3 und 4 muss berücksichtigt werden, dass hier auch eine Gefährdung durch holzzerstörende Insekten bestehen kann. Diese werden nicht zwingend durch dieselben $\mathrm{Mi}$ kroorganismen in ihrer Entwicklung gehemmt wie die holzzerstörenden Pilze. Schimmelpilze können je nach Art entweder durch Aufschluss der Holzsubstanz eine Insektenentwicklung fördern, durch Nahrungsentzug diese behindern oder durch Metaboliten toxisch auf Insekten wirken.

Ein interessanter Aspekt könnte die bekämpfende Behandlung von geschädigtem $\mathrm{Holz}$ sein mit dem Ziel, einen bestehenden Befall durch holzzerstörende Pilze in seiner Ausbreitung zu verzögern, um die Gebrauchsdauer eines Holzbauteils für eine bestimmte limitierte Zeit zu verlängern. Gelingt es hier ein antagonistisches Pilzgemisch mit einer Breitbandwirkung in einer hohen Dosierung ins $\mathrm{Hol} z$ einzubringen, dürfte eine längere Wirkung erzielt werden als durch Wirkstoffe mit einem hohen Dampfdruck.

Aus hygienischen und allergologischen Gründen dürfte der Einsatz von antagonistischen Mikroorganismen auf Holzbauteilen in Feuchträumen ausgeschlossen sein.

Bei der Evaluation der Wirksamkeit biologischer Verfahren in Laborversuchen ist wichtig, dass die Antagonisten stets gegen ein Pilzgemisch geprüft werden, wie es in der Praxis in der entsprechenden Gefährdungsklasse anzutreffen ist. Denn nur in dieser Konstellation kann nachgewiesen werden, ob der Nützling gegenüber der residenten Mikroflora bestehen und seine Wirkung entfalten kann. Prüfungen gegen einzelne Pilzstämme, wie sie in der europäischen Norm EN 113 vorgesehen sind, können keinen Aufschluss über deren Eignung geben. Im Unterschied zu den europäischen Normen müssen auch wesentlich längere Versuchszeiten eingeplant werden, damit festgestellt werden kann, ob durch die Nutzpilze langfristig nicht auch die Holzstruktur zerstört wird.

Eine weitere Option neben dem biologischen Holzschutz ist die biotechnologische Massenproduktion von antibiotischen Stoffen mit Hilfe genetisch optimierter Mikroorganismen. Da in der Regel diese Stoffe keine hohe Persistenz gegen die verschiedenen Umwelteinflüsse aufweisen, dürften sie fast ausschließlich für den temporären Holzschutz gegen Schnittholzbläue in Frage kommen, oder sie müssten mit einem Deckanstrich gegen die Witterungseinflüsse geschützt werden. 
Kritisch ist bei der biotechnologischen Herstellung vor allem die Extraktion der reinen Wirkstoffe. Denn für die human- und ökotoxikologische Prüfung müssen die Stoffe ohne Verunreinigungen durch andere chemische Verbindungen vorliegen, damit die pilzwidrigen Stoffe (Fungizide oder Fungistatika) bei positiver Beurteilung generell eingesetzt werden können. Nur eine politisch motivierte Gesetzgebung könnte hier den biogenen gegenüber den synthetisierten chemischen Stoffen eine Präferenz geben, indem an die beiden Stoffgruppen nicht dieselben Anforderungen bezüglich Human- und Ökotoxizität gestellt würden.

In jedem Fall dürften von Pflanzen und Mikroorganismen produzierte antimykotische Metaboliten in Zukunft an Bedeutung gewinnen. Hier liegt noch ein großes Potential, wenn es gemeinsam von Biologen, Chemikern und Toxikologen durch gezielte Forschung erschlossen wird. Diese biogenen Stoffe können als Modelle für die chemische Synthese persistenterer Verbindungen dienen. Als Beispiele können die gegen Holzinsekten wirksamen chemisch synthetisierten Pyrethroide angeführt werden, welche im umweltlabilen Pyrethrum von Chrysanthemen ihr Vorbild hatten.

Um im Holzschutz neue Wege beschreiten zu können, müssen die Schwerpunkte der Grundlagenforschung auf das Verständnis der Biologie der Schad- und Nutzorganismen, die Bedingungen und die Art der Kolonialiserung des Holzes, die verschiedenen potentiellen Schutzmechanismen in verschiedenen Holzarten und daraus folgernd auf die an die Gefährdung des Holzes angepassten optimalen Kombinationen von planerischen, konstruktiven, biologischen, biotechnologischen, biotechnischen und chemischen Maßnahmen und Verfahren gelegt werden. Die Nutzorganismen und ihre Wirkstoffe sind dabei nicht nur auf ihre antagonistische Wirkung gegen Pilze, sondern auch auf ihren möglichen Einsatz zur Erhöhung der Wegsamkeit des Holzes für Schutzmittel zu untersuchen. Ergibt sich aus den biologischen Versuchen, dass das Holz vor der Behandlung mit antagonistischen Mikroorganismen behandelt werden muss, so muss das Verfahren als praxisuntauglich und unökonomisch abgeschrieben werden. Ein weiterer Ansatz ist die Kombination von Mikroorganismen mit niedrigen Dosen von Fungiziden wie z.B. mit Fluor, Bor oder einem organischen Wirkstoff. Für den Nachweis der Wirksamkeit von antagonistischen Mikroorganismen sind neue Prüfmethoden zu schaffen, welche die verschiedenen Möglichkeiten von Interaktionen der Mikroflora innerhalb des Ökosystems Holz berücksichtigen.

\section{Literatur}

Baker R (1968) Mechanisms of biological control of soilborne pathogens. Ann. Rev. Phytopathology 6: 263-294
Bruce A, King B, Highley TL (1991) Decay resistance of wood removed from poles biologically treated with Trichoderma. Holzforschung 45: 307-311

Canessa EA, Morrell JJ (1996) Effect of Trichoderma harzianum on induction of laccase by Trametes versicolor on ponderosa pine sapwood. Intern. Res. Group Wood Pres. IRG/WP 96-10177, $13 \mathrm{~S}$ Carson R (1968) Der stumme Frühling. Deutscher Taschenbuch Verlag $\mathrm{GmbH}$, München: $346 \mathrm{~S}$. (englische Version: 1962) Franz JM, Krieg A (1972) Biologische Schädlingsbekämpfung. Verlag Paul Parey, Berlin, Hamburg: $208 \mathrm{~S}$

Freitag M, Morrell JJ, Bruce A (1991) Biological protection of wood: status and prospects. Biodeterioration Abstracts 5: 1-13 Giron MY, Morrell JJ (1989) Interactions between microfungi isolated form fumigant-treated Douglas-fir heartwood and Poria placenta and Poria carbonica. Material und Organismen 24: 39-49 Graf E (1990) Biologischer und biotechnischer Holzschutz - Literaturreview. Interner Bericht 120'500, EMPA St. Gallen, $29 \mathrm{~S}$ Graf E (1997) Bibliographie - Biologischer, biotechnologischer und biotechnischer Holzschutz. EMPA St. Galler.

Greaves H (1970) The effect of selected bacteria and actinomycetes on the decay capacity of some wood-rotting fungi. Material und Organismen 5: 265-279

Highley TL, Ricard J (1988) Antagonism of Trichoderma spp. and Gliocladium virens against wood decay fungi. Material und Organismen 23: 157-169

Kreber B, Morrell JJ (1993) Ability of selected bacterial and fungal bioprotectants to limit fungal stain in ponderosa pine sapwood. Wood Fiber Sci 25: 23-24

Kunz B, Weidenbörner M, Adams S, Strohmeyer M, Wüstenhöfer B, Wegen H-W (1995) Chancen und Möglichkeiten der Entwicklung umweltschonender Holzschutzmittel. In " 20 Holzschutz-Tagung 1995" (ed) Deutsche Gesellschaft für Holzforschung (DGfH), München 133-140

Liu L, Morrell JJ (1996) The role of chitinase in bioprotectant activity against wood staining fungi. Intern Res Group Wood Pres IRG/WP 96-10175, $11 \mathrm{~S}$

Messner K, Fleck V, Marchler A, Bürgel J, Horwath E, Schlick E (1995) Stand der technischen Entwicklung des biologischen Holzschutzverfahrens LCT. Berichtband der 20. DGfH-Holzschutztagung, DGfH München 111-119

Morrell JJ (1990) Effect of volatile chemicals on the ability of micro-fungi to arrest basidiomycetes. Material und Organismen 25: 267-274

Morrell JJ, Sexton CM (1992) Effect of nutrient regimen, temperature, $\mathrm{pH}$ and wood sterilization method on performance of selected bioprotectants against wood staining fungi. Intern Res Group Wood Pres IRG/WP 92-1551

Morrell JJ, Velicheti RK (1995) Effect of Pseudomonas cepacia on the activity of a mixture of wood staining fungi on poderosa pine sapwood. Intern Res Group Wood Pres IRG/WP 95-10107 Murmanis LL, Highley TL, Ricard J (1988) Hyphal interaction of Trichoderma harzianum and Trichoderma polysporum with wood decay fungi. Material und Organismen 23: 271-279

Oxley TA (1977) Report on biological control of decay in wood poles. Intern. Res. Group Wood Pres. IRG/WP 149

Schmidt O, Müller J (1996) Praxisversuche zum biologischen Schutz von Kiefernholz vor Schimmel und Schnittholzbiäue.

Holzforschung und Holzverwertung 81-84 